\title{
Les enfants de Lamech dans la Bible anonyme (BNF fr. 763)
}

\author{
Julia C. Szirmai
}

(C) The Author(s) 2011. This article is published with open access at Springerlink.com

\begin{abstract}
The thirteenth century Bible anonyme contains a story about the children of Lamech which, as far as I could ascertain, is unique amongst Old French bible texts. The story not only differs from that in Genesis 4: 19-22, but also deviates from corresponding passages in other Old French bible adaptations, which usually stress Lamech's role as the first bigamist and repeat the legend that it was Lamech who killed Cain. In the present text Lamech has ten sons, instead of the usual three; this may be the consequence of an incorrect interpretation of a commentary in the Glossa Ordinaria. What is even more remarkable, though, is the description of his daughter, Naamah, as a lascivious woman and the monologue she delivers. It is difficult to determine whether this portrayal is based on patristic sources or on Jewish ones. One interesting conclusion is that there appears to be an obvious connection (even textually) between the description of Naamah in the Bible anonyme and her appearance in the fifteenth century Mistère $d u$ Vieil Testament. Although it is virtually impossible to pinpoint the sources of the mystères, the assumption that there is a common source for both texts is an attractive one.
\end{abstract}

Keywords Bible anonyme Paris, BNF fr. 763 . Traductions-adaptations en vers de la Bible en ancien français - Mistère du Vieil Testament . Sources patristiques et juives

J. C. Szirmai $(\bowtie)$

Département de Françals, Universiteit Leiden, Opleiding Franse taal en cultuur,

Postbus 9515, 2300 RA Leiden, The Netherlands

e-mail: J.C.Szirmai@hum.leidenuniv.nl 


\section{La Bible anonyme Paris, BNF fr. 763}

La Bible anonyme ${ }^{1}$ (XIII $^{\mathrm{e}}$ siècle) est une traduction-adaptation de l'Ancien Testament, plus précisément de la Genèse, de l'Exode, des Nombres et de quelques emprunts au Lévitique et au Deutéronome. Cette Bible présente ensuite une version assez complète de la légende de la Croix, ${ }^{2}$ où est incorporée la Passion du Christ.

L'auteur suit assez fidèlement la Vulgate, qu'il prétend raconter se [de] la memoire dou cuer non (v.5341) ${ }^{3}$ pourtant il lui arrive de combiner des épisodes et d'ajouter des détails qu'on ne lit pas dans la Bible. ${ }^{4}$ À part la légende de la Croix, la Bible présente d'autres légendes, que l'auteur mêle parfois avec l'histoire biblique: ainsi, aux vv. 2551-2564, il raconte que Joseph est vendu au senechaut lou roy Pharaon, tandis que c'est la reine qui s'éprend de lui ${ }^{5}$ bien que l'auteur, pour plusieurs de ces histoires, se base sur l'Historia Scholastica [désormais HS] de Petrus Comestor, il semble ne savoir que faire de la légende, bien connue, du charbon ardent qui brûle la langue du petit Moïse. ${ }^{6}$

Pour ses expansions l'auteur met à profit des sources patristiques et juives ou un mélange des deux: ainsi, relatant 'l'estoutie' de Ruben, il dit: Ce ne fu pas escript, mas ce est prophecie/Que apartient as Juis (...), ${ }^{7}$ vers pour lesquels il semble se baser aussi bien sur saint Jérôme que sur saint Isidore, cités par la Glossa ordinaria. ${ }^{8}$

Si l'auteur de la Bible anonyme ne se distingue pas d'autres adaptateurs de textes bibliques en introduisant des éléments apocryphes et légendaires dans son adaptation, il est le seul, pour autant que je sache, à développer le récit des enfants de Lamech.

L'histoire de Lamech, descendant de Caïn, ${ }^{9}$ se trouve dans la Genèse IV, $18-24:^{10}$

‘... et Mathusael genuit Lamech. Qui accepit duas uxores: nomen uni Ada, et nomen alteri Sella. Genuitque Ada Jabel, qui fuit pater habitantium in tentoriis, atque pastorum. Et nomen fratris ejus Jubal; ipse fuit pater canentium

\footnotetext{
1 Szirmai (1985).

2 Szirmai (1985), vv. 7140 ss. Voir aussi chap. II.6.c.

3 Bien qu'il prétende aussi disposer d'une version écrite: [...] mansions/Don je truis en versez les noms (4754-4755); ne l'escrist point nou monstre (3783).

4 Ainsi le fait que le peuple juif peut passer par la Mer Rouge grâce au fait que icele nuit gela et que la mer fu comme murs (...)/Dou giel et de la glace (vv. 4631-4635).

5 De même dans Li Romanz de Dieu et de sa Mere d'Herman de Valenciennes, dans la Bible des sept estaz du monde de Geufroi de Paris, dans la Bible de Jehan Malkaraume et dans les Anglo-norman Bible stories, où l'auteur nous explique la confusion ainsi: Les marchauntz (...) le vendirent a Putifar, le seneschal le roy Pharaon, e il dona pur sa belté a le roy (...). .

6 Vv. 3781-3783. HS, Patrologia Latina [PL] 198, 1144 A/B.

7 Vv. 2339-2340.

${ }^{8}$ Gibson (1992, Gen., p. 107).

9 À distinguer du père de Noé, épisodes confondus dans beaucoup de textes médiévaux. Voir aussi Murdoch (2003) et Reiss (1972).

${ }^{10}$ Biblia sacra iuxta Vulgatam clementinam (Colunga 1982).
} 
cithara et organo. Sella quoque genuit Tubalcain, qui fuit malleator et faber in cuncta opera aeris et ferri. Soror vero Tubalcain, Noema'.

La Vulgate nous apprend encore qu'il avoua à ses femmes avoir tué un homme ‘in vulnus meum' et un jeune homme 'in livorem meum' (Gen. IV, 23).

\section{Les traductions-adaptations bibliques françaises ${ }^{11}$}

Les traductions-adaptations bibliques en ancien français reprennent, pour la plupart, les faits de la Genèse, sauf celles de Malkaraume et de Herman de Valenciennes, qui passent sous silence le passage entier. L'histoire de Lamech manque dans la Bible des sept estaz du monde de Geufroi de Paris. ${ }^{12}$

\section{Les femmes de Lamech}

Dans la plupart des textes bibliques c'est par la bigamie de Lamech que débute l'histoire de ses enfants. Ainsi dans la Genèse d'Evrat [Evr]: ${ }^{13}$

\section{Lamech (...)}

1536 Dous femmes prist; bien vos dirai

Les nons, puis c'al dire m'essai:

L'une fu apelee Ada

Et l'altre avoit a non Sella

dans la Bible de Macé de La Charité [MC]: ${ }^{14}$ Cil ot.ij. fames, ceu me semble,/ Sella et Oda tout ensemble, ou dans le Poeme anglo-normand [Pan]: ${ }^{15}$ Dous femmes prist Lamech (...), la Bible du XIIIe siècle [BXIII]: ${ }^{16}$ (...) Lamech qui prist.II. fames: l'une ot non Ada, et l'autre Sella et la Bible d'Acre [BdA]: ${ }^{17}$ De sa lignee fu Lamech qui ot.II. femes.

Dans la Bible de Macé la bigamie de Lamech est explicitement nommée: Et ce fu li premers bigames/Que tout ensemble ot.ij. fames; (vv. 669-70); ${ }^{18}$ dans les

\footnotetext{
11 Pour faciliter la lecture de cet article, nous donnons ici les sigles représentant les textes bibliques cités: [Evr] la Genèse d'Evrat; [MC]: la Bible de Macé de La Charité; [Pan]: Poème anglo-normand sur l'Ancien Testament; BXIII]: la Bible du XIIIe siècle; [BdA]: la Bible d'Acre; [HL] Histoires tirées de l'Ancien Testament; [BH]: Guiart Des Moulins, Bible historiale; [MVT]: Mistère du Vieil Testament; [M]: Montpellier, Bibliothèque de l'École de Médecine, f. fr. 437 et [A]: Paris, Bibliothèque de l'Arsenal, f. fr. 3516 .

12 Dans le ms BNF fr. 1526, contenant la Bible de Geufroi de Paris, il manque un folio entre les feuillets 13 et 14

13 Boers (2002). Il est intéressant de noter qu'Evrat qui fait un usage abondant des gloses dans sa Genèse, et qui ne dédaigne pas les diatribes morales, ne s'arrête pas sur le péché de Lamech.

14 Smeets (1967, I, vv. 667-668).

15 Nobel (1996, I, v. 198).

16 Quereuil (1988, p. 124).

17 Nobel (2006, p. 10, 18).

18 Voir aussi Petrus Riga, Aurora, Liber Genesis 462 (Beichner 1965). Voir Smeets (1967) I, p. 29, n. 709-745 et. Murdoch (2003, p. 72, p. 76). La bigamie de Lamech figure aussi dans le Livre de Leesce de
} 
Histoires tirées de l'Ancien Testament $[\mathrm{HL}]^{19}$ Lamech est condamné par l'auteur: et fu tres mauvais et li premiers bigames.

Guiart Des Moulins $[\mathrm{BH}]^{20}$ ayant dit: Lamech prist.ii. femes: li une eut a non Ade et li autre Sella (XV, 4-5), ajoute dans son commentaire:

(...) Lamech qui fu li.vii. ${ }^{\text {me }}$ du lignage Adan et tres mauvais, car il amena avant premiés bigamie et eut.ii. femes; et fist ausi adultre encontre le loy de nature et encontre l'establissement de Dieu. Car en le premiere creation des hommes fu une seule feme donee a.i. seul honme, si conme Eve a Adan; et Dieus ensi avoit establi par le bouche Adam que li hom et li feme en mariage seroient doi en une char.

Le Mistère du Vieil Testament $[\mathrm{MVT}]^{21}$ prête la parole à Lamech qui se vante de cette nouveauté dans les relations humaines:

Je ne suys pas content d'Adam,

Qui dit que c'est divine loy

Que ung homme n'ayt avec soi

3350 Que une femme; c'est grant follye;

Introduyre vueil bigamye

Et contre cest esdit aller;

Deux femmes je veulx acoller,

Car en effect j'en ay pou d'une

La bigamie de Lamech- et ce n'est pas pour nous étonner- a fait l'objet de plusieurs commentaires patristiques: ainsi celui de saint Jérôme: 'Primus Lamech sanguinarius et homicida, unam carnem in duas divisit uxores'. ${ }^{22}$ La Glossa ordinaria affirme: 'In hac progenie Lamech primus contra morem, contra naturam, per bigamiam adulterium commisit'. ${ }^{23}$ Petrus Comestor, dans l'Historia Scholastica, dit de Lamech: '(...) qui primus bigamiam introduxit, et sic adulterium contra legem naturae, et Dei decretum, commisit'. ${ }^{24}$

Si Flavius Josèphe relate seulement le fait que Lamech avait deux femmes, la tradition juive, elle aussi, met l'accent sur le péché de Lamech, tout en suggérant qu'il n'etait pas le seul bigamiste de son époque : 'The corruptness of the times, and

\section{Footnote 18 continued}

Jean Le Fèvre, une traduction des Lamentationes de Mathéolus datant du XIVe siècle. Voir A.-G. van Hamel (1905), tome 2, vv. 92 ss. La 'bigamie' s'applique ici également à l'homme qui épouse une veuve, ou à l'homme qui séduit une femme mariée.

19 Loh (1912, p. 8, 15).

${ }^{20}$ Michel (2004), XV, 23-28. Le poète néerlandais, Jacob van Maerlant, s'exprime en des termes plus forts: 'Lamech/die was een quaet man/ Dit was die zevende van Adam/Entie eerste die.ij. wijf nam./Dus dede hi hoerdoem an die wet;/ want God hadde int eerste gheset/Dat si.ij./een man en een wijf/waren een vleesch en een lijf./ Hine seide niet:.j. man nemer twee,/Lamech achtets min no mēe' (Rymbybel I, vv. 960-968. (David 1858)).

21 Rothschild (1878-1891), vv. 3347-3354.

22 PL 23 (Adversus Jovinianum 233).

23 Gen., p. 33.

24 Sylwan (2005), 1078C. 
especially the depravity of Cain's stock, appears in the fact that Lamech, as well as all the men in the generation of the deluge, married two wives, one with the purpose of rearing children, the other in order to pursue carnal indulgences (...) ${ }^{25}$

\section{Le nombre d' enfants de Lamech}

Selon la Genèse (IV, 20-22) Lamech a trois fils: Jabel, Jubal et Tubalcain et une fille Noema.

Bien que Flavius Josèphe prête à Lamech 77 enfants, ${ }^{26}$ et que [Pan] ne mentionne que Tubalchaim et Jubal, ${ }^{27}$ les traductions bibliques françaises suivent pour la plupart la Vulgate en lui attribuant trois fils, l'auteur de [BdA] étant le seul à ne pas leur donner de noms.

Par contre, leur soeur Noemie [Noema] n'est nommée que par Evrat qui attribue à Tubalcain plusieurs soeurs: 'Une de ses serors i a/Ki ot a nom Neomïa' (1560), par la Bible du XIIIe siècle: 'La suer Thubalchaym ot not (sic) Noema' (p. 124), dans le commentaire de la Bible de Guiart des Moulins II, 556/58: 'Noema, li suer de Thubalchaym' et dans [MVT], vv. 8360-8520.

\section{Les occupations des fils}

Jabel, qui fuit pater habitantium in tentoriis, atque pastorum. Et nomen fratris ejus Jubal; ipse fuit pater canentium cithara et organo. Sella quoque genuit Tubalcain, qui fuit malleator et faber in cuncta opera aeris et ferri (Gn IV, 19-22).

Avec plus ou moins de commentaires, les auteurs des adaptations bibliques prêtent aux enfants de Lamech les occupations qu'il remplissent selon la Genèse:

[Evr]: (...) Gebal,/

En lui ot mervilhous vasal, K'il habita premiers en tentes, La mist il totes ses ententes,

1544 Et si fu des pastorelz mestre,

$\mathrm{Ki}$ lor bestes menoient pestre

Et Jubal rot a nom ses frere,

Nel wel de mon parcemin rere,

1548 K'il sot et de l'orgene et del chant,

Encor nel lais je mie a tant:

Ce fu li promiers orgenere

Et de la harpe bons menere

\footnotetext{
$\overline{25}$ Ginzberg (1911-1938), I, p. 117. Voir aussi Reiss (1972, pp. 40-41).

26 Antiquités juives, I,2,2 (Blatt 1958).

27 Écart du texte biblique non commenté par l'éditeur moderne.
} 
(...)

(...) Tubalchaÿn,

Cil [fu] wimes aprés Chaÿn;

1556 Fevres fu cil trop mervilhous,

N'estoit pas d'ovrer orguilhous,

K'en oevre de fer et d'arain

Le tenons nos a premerain

Selon [MC] Jabel et Jubal sont tous les deux des pasteurs (vv. 672-674). Jubal est en plus

En estrumens d'orgue ententis,

Organistres fu et herperres

Et de musique fu troverres. (vv. 676-678)

L'origine de son métier étant le travail de son frère Tubalcaïn qui

(...) fu li premiers qui seüst

Comment l'on fer fere deüst,

Et de toz metaux la mestrise

Fu par lui trovee et aprise; (vv. 695-698)

car:

$\mathrm{Au}$ cos que sur les metaux fist

Jubal musique a fere emprist;

Li cob qui sur metal sonaint

Aucune acordence donaint

Et pour cetui son Jubal prist

L'escort qu'il en musique mist;

Enssi fu musique establie (vv. 699-705) ${ }^{28}$

Comparons les métiers des frères dans:

[Pan]:

Jubal truva primes e harpe e chant

E estrumenz plusurs a son talant

Li premer frere ceo fut Tubalchaim,

Cist trova de tuz metals engin. (vv. 200-204)

[BdA]: li uns fu habiteors en tentes et pastre, l'autre fu chanteor en chiterelle et en orgres, li tiers fu fevres de toz labors d'arrain et de fer. (...) (p. 10, 19-21). ${ }^{29}$

[BXIII]: Yabel qui fu peres de ceuls qui habitoient es tabernacles, et iert des pasteurs; et li nons de son frere est Jubal: cil fu peres de ceuls qui chantoient en orgue. Et Sella engendra Tubalchaym qui fu maillierres et fevres de toutes oevres d'arain et de fer (p. 124).

${ }^{28}$ Voir HS, Sylwan (2005), 1079; voir aussi [BH].

${ }^{29}$ Dans la glose on lit: *** En ces mist Dieu premierement divers mestiers. 
$[\mathrm{BH}]$ : Jabel qui trouva premierement les coses que li pasteur portent, si conme pars de berbis et tentes et autres coses pour muer pastures; et ordena les brebis par fous et les enseigna; et departi les brebis des bous et des kievres; et mist ensamble, par eles, les berbis d'une couleur et tavelees ausi par eles; et cheles d'un an et les aigniaus departi des aisnees; et perchut le conjunction des berbis a faire en certain tans. Ses freres Jubal fu peres des cantans et harpes et en orghenes, ne mie des estrumens qui ore sont, car il furent lonc tans aprés trouvé; mais il trouva musike et acordanches, par quoi li travaus des pasteurs fust tournés en plaisanche et en delit.(....). ${ }^{30}$ (...) Thubalcaym qui trouva premiers l'art d'ouvrage de fer et les armeures a combatre ${ }^{31}$ et les entalleures de dyvers ouvrages en metaus et les delitables coses a veir. Et en eles favrecant de ses marteaus, li devant dis Jubal, qui fist l'art de musike, se delita el son des marteaus: si fist et pourpensa, par les pois des marteaus, les consonancies et les proportions et les acordanches qui ore viennent des divers pois des marteaus, si conme en orloges et en aucuns autres estrumens (pp. 554-556). ${ }^{32}$

[HL]: (...) Jabel qui trouva premiers l'ordenanche du mestier de bregerie; (...) Jubal qui premiers trouva l'art d'organerie et de consonancie de musique (...). Tubacaym qui fu li premiers fevres (pp. 8-9)

[MVT]:

Jabel a donné cognoissances/de departir brebis, aigneaux/et les acoutrer par troupeaux;/(...), et moy [=Jubal], j'ay trouvé la praticque,/l'art et science de musique,/qui est plaisante a escouter.(...) Vous [=Tubal] avez trouvé sans debatre/ l'art d'armeures pour gens combatre,/de faire plusieurs instrumens/de fer pour les esbatemens/des humains (...). (vv. 5761-5778).

\section{Noemie}

Si la soeur des trois frères est nommée, le rôle de Noemie est passé sous silence dans la Vulgate $(\mathrm{Gn} I \mathrm{~V}, 22)$ et dans les traductions bibliques, sauf dans [BH]: Noema, li suer de Thubalchaym, trouva l'art de tistre diversement, et de divers ouvrages (p. 558) et dans [MVT]: Noema, nostre seur germains, / trouva l'art de tixture en laine (vv. 5766-5767). On trouve la source de cette assertion dans l'Historia Scholastica. ${ }^{33}$ Les Pères et les légendes juives (et l'auteur de [MVT]) prêtent à Noemie encore d'autres qualités, comme on le verra par la suite.

\footnotetext{
${ }^{30}$ Voir HS, 1079; Aurora (Beichner 1965), 468. Curieusement cette dernière observation manque dans [MC].

31 Voir Antiquités juives. I,2,2: 'Jobel autem (...) fortitudine cunctos excellens res bellicas decenter exercuit'. Voir aussi HS, 1079 et Aurora, 478. Comparez [MTV] v. 5775.

32 Nous laissons de côté la légende des deux colonnes sur lesquelles Jubal 'escrist (...) l'art de musike qu'il avoit trouvé (qu'ele ne fust perie par ewe ne par feu) (...) ([BH] p. 556, 40-41), légende qu'on retrouve dans quelques adaptations bibliques. Voir aussi $H S, 1079$.

33 Sylwan (2005), 1079 : 'Soror vero Tubalcain, Noema, que invenit artem varie texture'. Pas de détails dans les Ant. Voir aussi Fillion I(1923), note ad Gn IV, 22.
} 


\section{Lamech tue un homme}

Dans Gn IV, 23 on lit:

Dixitque Lamech uxoribus suis Adea et Sellae: Audite vocem meam, uxores Lamech, auscultate sermonem meum: quoniam occidi virum in vulnus meum; et adolescentulum in livorem meum. 24. Septuplum ultio dabitur de Cain; de Lamech vero septuagies septies.

À part [Pan] et [HL] qui ne parlent pas de cet épisode, la plupart des textes bibliques identifient le virum biblique avec Caim ([Evr] 1606, 1616; [MC] 712; [BdA] p. 10, 21-22; [BXIII] p. 125; [BH] p. 558,70), d'après l'histoire donnée par Comestor: 'casu interfecit Cain inter frutecta, estimans feram. Quem quia ad indicium juvenis dirigens sagittam, interfecit'. 34

L'adolescentulum' reste anonyme ([Evr]: l'enfant 1618 (voir aussi [BH] 558), [MC]: le vaslet 735, [BXIII]: un jouvencel p. 124), ${ }^{35}$ sauf dans [MVT] où Lamech tue son propre fils Tubalcaim (4785). ${ }^{36}$

Comme on a pu le constater, les traductions bibliques en ancien français suivent assez fidèlement l'histoire de la Vulgate. Pour quelques détails elles puisent dans l'Historia Scholastica qui se base parfois sur des traditions juives.

L'histoire de Lamech dans la Bible anonyme ${ }^{37}$ s'éloigne d'une manière singulière de celle que l'on trouve dans les autres textes. Le récit se présente ainsi:

$X$. en $\mathrm{i}$ ot et une fille,

724 De ceus en issi bien.iij. mile

Des nevous conterons l'estoire

Que devons havoir en memoire

Por eschuïr lour felonie

728 Et garder de lur compaignie

Li uns d'aus aprit a forgier

Et fist armes por guerroier

Li autres, charpentiers igniaus,

732 Bertoiches fist et mangonniaus,

Et li tiers d'aus fuit oiselerres ${ }^{38}$

Et li quars fuit merveillous lierres

Li quins m'est vis qu'il fu chacierres ${ }^{39}$

\footnotetext{
34 Sylwan (2005), 1079. Pour plus de détails sur cette légende voir Smeets, Bible de Macé I, pp. 31-32, Dictionnaire des Mystères (Douhet 1854, 1007-1008), Murdoch (2003, p. 74ss)., E. Reiss (1972, pp. 30-40), Mellinkoff (1981) et J.H. Morey (1993, pp. 13-15).

35 [BdA] ne parle que de Caim (p. 10, 21-22).

36 Voir Murdoch (2003, pp. 72-74).

37 Les variantes de la Bible anonyme, Montpellier, Bibliothèque de l'École de Médecine, f. fr. 437 [M] et Paris, Bibliothèque de l'Arsenal, f. fr. 3516 [A] présentent pour la plupart du fragment le même récit; il est intéressant de noter que [M] ajoute plus tard la version canonique de la Genèse.

38 [A] 726-748 om.

$39[M]$ peschierres.
} 
736 Et li sixaimes fu archierres ${ }^{40}$

Li septaimmes fuit fors et fiers, ${ }^{41}$

$\mathrm{Li}$ octeimmes fuit chevaliers

Li noneimmes torna son san

740 A soner divers estrumans:

Tymbre, harpe, trompe, viele, ${ }^{42}$

Sarterion et camponnele ${ }^{43}$

Li dismes fuit clers en s'anfance

744 Et aprist l'art de nigromance;

Les regles en sout et les pars

Et sout chasconne des.vij. ars:

Gramaire aprit por droit parller,

748 Dealitique pour monstrer

La verité et la mançonge

De raison ou il a chalonge

Et rectorique, que est sens

752 D'ordener les drois jugemans

Et les raisons et les paroles

Que on aprant par les escoles;

De nombre fist arismetique

756 Et de voiz ordena musique,

De mesure geometrie,

Des estoilles astronomie:

Quel nom chasconne a et quel cors

760 Et quel croissant et quel decors

L'onzaime fuit une donsele,

Auques fole, mais mult fuit bele

De nul bien n'estoit enseignie,

764 Touz jours estoit et baude et lie;

N'ot en li nulle contenance, ${ }^{44}$

Mult fist folies en s'anfance

Trop estoit en mal vezïe $e^{45}$

768 Et de respondre enseigniee;

A chascun scevoit raison rendre,

$\mathrm{Ne}$ la pouest nuns entreprandre,

Et de sa folie escuser

772 Ne veïstes onques sa per

Onques de riens ne s'amanda,

Nul n'escondi qui la pria

\footnotetext{
${ }^{40}[M]$ chacierres.

$41[M]$ archiers.

${ }^{42}[M]$ gighes, harpes, rotes, violes.

$43[M]$ soner corron et campenoles.

$44[\mathrm{M}][\mathrm{A}]$ astenance.

$45[\mathrm{M}]$ t. Par savoit mal et voisdie.
} 
Itant disoit que nulle famme

776 Ne doit por son cors perdre s'ame:

'Famme fu a home donee,

Cele est fole qui son cors vee;

Por ce ai je nom Noëmie

780 Que ja nul home n'escondie;

Se je fais a home son gré,

Je ne cuit riens meffaire a Dieu;

Je suis preste si com je doi,

784 Hons se gart de faire desroi'

Ouez de la fole cheitive

Com s'estoit livree gaïve

Lamec, ce m'est vis, fuit ses peres

788 Et por li ocist un suen frere

A ses.ij. fammes s'en vanta

Qu'il l'avoit mort, sel compara

Le fragment présente plusieurs particularités qui ne se trouvent pas dans la Genèse:

(a) les deux femmes de Lamech n'ont pas de nom

(b) la liste que présente la Bible anonyme des 'nevous' de Caïn, les enfants de Lamech, comprend dix fils et une fille; à part la fille, les enfants n'ont pas de nom

(c) les occupations des fils diffèrent de celles mentionnées dans la Genèse; Tubalcain, Jubal et Jabel ne sont pas mentionnés comme inventeurs

(d) l'auteur anonyme consacre 28 vers au personnage de Noemie, dont le rôle est passé sous silence dans la Vulgate

(e) le vers 788 semble reprendre la légende selon laquelle Lamech tue Tubalcain; il n'est pas question du meurtre de Caïm.

Regardons de plus près ces particularités

\section{(a) Les femmes de Lamech}

L'histoire des enfants de Lamech est introduite, dans la Bible anonyme, par un récit tant soit peu confus sur les descendants de Caïn. En parlant des fils d'Hénoch, l'auteur nous dit:

Li un vivoient de rapines

Et li autre de sauvaigines

Et li autre de larrecin:

Touz les doutoient li veisin, (vv. 719-722)

après quoi suit notre vers 723: ' $\mathrm{X}$ en $\mathrm{i}$ ot et une fille'. Bien que l'auteur semble établir un rapport direct entre les fils d'Hénoch et les enfants de Lamech, il n'est pas impossible qu'il manque un ou plusieurs vers après 722; ce n'est qu'au vers 787 
qu'on apprend que les ' $\mathrm{X}$ ' 'et une fille' sont des enfants de Lamech. Le ton négatif de sa présentation des descendants de Lamech (727-728) n'est pas seulement une réminiscence de Gn VI, 1-5 où 'la malice des hommes' est attribuée à l'union des 'filii Dei' (race de Seth) avec les 'filias hominum' (race maudite de Caïn); l'impiété des descendants de Caïn est développée par Flave Josèphe qui dit par rapport à Caïn:

'(...) licet cum iniuria secum commorantium hanc haberet. Augens domum multitudine pecuniarum, ex rapinis et violentia congestarum, ad luxuriam quoque et latrocinia suos familiares invitans, quorum doctor in scelestis studiis erat $(\ldots)^{46}$

et dans la légende juive, ${ }^{47}$ qui attribue à Hénoch, fils de Caïn, les crimes dont notre auteur accuse les fils de celui-ci (ou de Lamech?):

He sinned in order to secure his own pleasure, though his neighbors suffered injury thereby. He augmented his household substance by rapine and violence; he excites his acquaintances to procure pleasures and spoils by robbery (...). ${ }^{48}$

L'auteur de notre texte précise aux vers 725-728 qu'il raconte l'histoire des fils de Lamech 'por eschuir lour felonie/et garder de lur compaignie'. Ce n'est qu'au vers 789 seulement que l'auteur parle des deux femmes de Lamech, qui restent dépourvues de nom dans notre texte. ${ }^{49}$ Il est remarquable que l'auteur anonyme n'insiste pas sur la bigamie de Lamech, fait que relatent les autres textes avec un certain sens du drame.

\section{(b) Le nombre d' enfants de Lamech}

Selon notre vers 723 Lamech a onze enfants, dix fils et une fille, nombre que l'on ne retrouve pas dans la tradition juive. Il semble que notre auteur a mal interprété les sources patristiques qui fournissent l'explication suivante du nombre onze:

Notandum quod generationes ab Adam per Cain undenario terminantur, in quo transgressio signatur. Lamech namque septimus est, cui tres filii et una filia adduntur, ut undenarius impleatur, per quem peccatum significatur: nam et a femina clauditur generatio, quae initium peccati fuit, per quod voluptas carnis, quae resistit spiritui. ${ }^{50}$

\footnotetext{
46 Antiquités juives, I, 2, 2. Voir aussi Muir (1995, pp. 72-73).

47 Ginzberg I, pp. 115-118; 121; 124 ss. Voir aussi Murdoch (2003, p. 76) et [MVT] où Seth dit à Caïn: 'Et bref tu ne mesleras point/ton lignage avec le mien' (vv. 4446-4447). Voir aussi J.-Cl. Schmitt (1988), I, 4.

48 Ginzberg I, p. 116. Il faudra donc corriger la traduction du mot 'sauvaigine' dans le glossaire de notre édition de la Bible anonyme en 'violence'.Voir aussi HS, 1078D, Smeets, Macé I, pp. 27-28, [BH] p. 554 (glose) et [MTV] vv. 3290 ss. Rien dans [Evr], [Pan], [BXIII], [HL], [BdA].

49 De même dans [Pan] et [BdA].

50 Glossa Ordinaria, Gen., p. 33).Voir aussi. Raban Maur, Comment. In Gen., PL 107, 510, saint Isidore, Quaest. in Vet. Test., PL 83, 229 et saint Augustin, De Civitate Dei, Lib.XVI, 6 (Ed. S. Santamarta del Rio y M. Fuertes Lanero, Madrid 1978).
} 
Comestor affirme que Lamech est 'septimus ab Adam', ${ }^{51}$ mais ne fait pas l'addition des quatre enfants de Lamech qui conduit au nombre du péché. Nulle part dans les traductions-adaptations en ancien français nous n'avons trouvé trace de cette exégèse.

\section{(c) Les occupations des fils}

Les occupations des fils de Lamech diffèrent de celles mentionnées dans la Genèse; Jabel, Jubal et Tubalcain ne sont pas mentionnés comme inventeurs.

Ce qui frappe d'abord dans notre texte c'est que les fils de Lamech, contrairement à la plupart des traductions bibliques, n'ont pas de nom; ils sont simplements indiqués par un nombre ordinal.

Nous reconnaissons dans le premier fils Tubalcaïn, l'inventeur de la métallurgie, qui de plus, selon notre texte, 'fist armes por guerroier' (v. 730), une assertion dont on trouve la source dans les Antiquités de Josèphe: 'Iobel autem qui ex altera natus est [=Sella]fortitudine cunctos excellens res bellicas decenter exercuit' et qui est reprise par $[\mathrm{BH}]^{52}$ et $[\mathrm{MVT}]$ :

Vous [=Tubal] avez trouvé sans debatre

l'art d'armeures pour gens combatre,

de faire plusieurs instrumens

de fer pour les esbatemens

des humains (...). (vv. 5761-5778).

Selon la tradition juive, 'Tubal-cain was rightly named, for he completed the work of his ancestor Cain. Cain committed murder, and Tubal-cain, the first who knew how to sharpen iron and copper, furnished the instruments used in wars and combats'.53

Le neuvième fils semble renvoyer à Jubal, le 'pater canentium cithara et organo'(Gn IV, 21), bien qu'il ne soit pas fait mention de l'orgue dans notre texte et que le neuvième fils joue encore plusieurs autres instruments. ${ }^{54}$

Pas de trace pourtant de Jabel, 'pater habitantium in tentoriis, atque pastores' (Gn IV,20).

Les métiers des autres fils sont apparemment une invention de l'auteur: le deuxième est charpentier, qui fait des bretèches et des machines de guerre. Le troisième est chasseur d'oiseaux, le quatrième, victime peut-être des exigences de la rime, est larron $^{55}$; le cinquième et le sixième sont respectivement chasseur ${ }^{56}$ et

\footnotetext{
51 Sylwan (2005), 1078. Dans quelques versions des Historienbibeln allemandes, Lamech a encore un fils. Voir Murdoch (2003, pp. 79-80).

52 Aniquités juives I,2,2. Voir aussi Aurora, 478, HS, 1079, Glossa Ordinaria, p. 33) et [BH], p. 556.

53 Ginzberg I, 117-118. Voir aussi Murdoch (2003, p. 93) et C.J. Verduin (2003), La recherche de GIIOHARGIIIVS, 'Tubalcain, Jubal \& Pythagoras'.

54 Voir [Evr]: K'il sot et de l'orgene et del chant (v. 1548); [PAN]: Jubal truva primes e harpe e chant/ E estrumenz plusurs a son talant, vv 200-201; [BXIII]: cil fu peres de ceuls qui chantoient en orgue, p. 124; $[\mathrm{BH}]$ : Ses freres Jubal fu peres des cantans et harpes et en orghenes, p. 556. Voir aussi Murdoch (2003), p. 91.

55 Pour un cas pareil, voir le Roman de la Rose, vv. 21.495-496.

56 Dans [M]: pechierres.
} 
archier, ${ }^{57}$ métiers traditionnellement attribués à Lamech, le septième est 'fors et fiers', ${ }^{58}$ le huitième chevalier. Le dixième fils est clerc et maîtrise non seulement les sept arts libéraux traditionnels: grammaire, dialectique, rhétorique (le trivium), arithmétique, musique, géométrie et astronomie (le quadrivium), mais aussi la 'nigromance', science qui est associée aux sept arts dans beaucoup de textes médiévaux. ${ }^{59}$

\section{(d) Noemie}

L'auteur de la Bible anonyme consacre 28 vers au personnage de Noemie. Il la décrit comme étant un peu folle, mais aussi très belle, vouée au mal, sans maîtrise de soi et sans aucune disposition à s'amender. Elle est, en plus, une putain qui ne refuse aucun homme, comme elle l'affirme elle-même dans un monologue provocateur de huit vers. Il est curieux de constater que l'auteur exhorte ensuite ses lecteurs à écouter l'histoire de cette 'cheitive' qui s'est livrée à la prostitution, ${ }^{60}$ sans pourtant leur donner ce récit. $^{61}$

$\mathrm{Si}$, dans les généalogies bibliques les filles sont rarement indiquées par un nom, ${ }^{62}$ dans la Genèse, la soeur de Tubalcain est explicitement nommée Noemie (Noema Gn IV, 22, hébreu: Naamah 'douce' ${ }^{\text {63 }}$ ); aussi son personnage a-t-il suscité des commentaires exégétiques.

Par rapport aux onze enfants de Lamech, nous avons vu que les exégètes expliquent ce nombre par l'addition d'une fille aux dix fils, saint Augustin précisant que le nombre dix représente la loi. ${ }^{64}$ La transgression de la loi est donc figurée par la soeur:

Lamech namque septimus est, cui tres filii et una filia adduntur, ut undenarius impleatur, per quem peccatum significatur: nam et a femina clauditur generatio, quae initium peccati fuit, per quod voluptas carnis, quae resistit spiritui. Unde Noema vocatur, id est, voluptas. ${ }^{65}$

\footnotetext{
57 Dans [M]: chacierres. Voir [MVT] 3777-8: 'La maniere de faire ung arc/J'ay trouvée; (...)'.

58 Allusion aux Antiquités juives I,2.2: 'decenter'?

59 Ainsi Roman de Rou, Roman de Troie (vv. 1217-1222), Partonopeu (vv. 4581-4598), Chronique de Philippe Mousket, vv. 9690-9801, Bataille des VII Arts, vv. 130-145. Dans le dernier texte nigremance est considérée comme l'aide magique de l'astrologie. Voir aussi L. Harf-Lancner, Les Fées au Moyen $\hat{A g e}$, Paris 1984, pp. 411-420. Voir A. van der Mark (2005, pp. 71-73).

${ }^{60}$ L'interprétation de gaïve reste difficile. Godefroy (IV, 203 donne comme interprétation de gaif: 'égaré' et 'vagabond'. Tobler-Lommatzsch met en rapport le mot gaï̀e avec gaigaie s.f. Dirne, Hure (IV, 40) ce qui semble être plus à propos.

61 Il n'est pas impossible qu'il manque un ou plusieurs vers après 786.

62 Saint Isidore, Quast. In Vet. Test., PL 83, 227C.

63 F.Vigouroux (1943), Dictionnaire de la Bible. Voir aussi L.-Cl.Fillion (1921-24) ad Gn, IV,22.

64 De Civitate Dei, XV, 20.

65 Glossa Ordinaria, p. 33: 'que interpretatur voluptas in qua significatus carnis concupiscentia'. Voir aussi saint Isidore, Quaest. in Vet. Test., PL 83, 229; saint Augustin, loc.cit.
} 
L'HS ne reprend pas ce commentaire et se contente de dire: 'Soror vero Tubalcain, Noema, quae invenit artem variae texturae'. ${ }^{66}$ Comestor suit, dans ce commentaire, la tradition juive. ${ }^{67}$ Les légendes juives, pourtant, ne sont pas toutes favorables à l'égard de la fille de Lamech. Ainsi, elle est parfois identifiée avec Lilith, la première femme d'Adam, esprit maléfique qui engendre des démons et qui étrangle des petits enfants. ${ }^{68}$ De plus, Naamah (=Lilith) 'fools men in their dreams' ${ }^{69}$ Regardons ce qu'en dit Ginzberg dans son Legends of the Jews:

selon la tradition juive, ${ }^{70}$ les descendants d'Hénoch (de la race de Caïn) sont les premiers idolâtres et "Naamah, "the lovely", earned her name from the sweet sounds which she drew from her cymbals when she called the worshippers to pay homage to idols'. ${ }^{71}$

'Naamah, Tubal-cain's sister, is further identfied with the beautiful woman to whose charms the angels became victims'. ${ }^{72}$ Ainsi, elle aurait séduit l'ange déchu Azazal et ' (...) in the kabbalistic sources (...) Naamah, the sister of Tubal-cain, is said to be the wife of Shamdan, from whose union sprang forth Ashmedai (=the devil par excellence), who together with Lilith strangles little children (...). ${ }^{73}$ 'Unlike Istehar, the pious maiden, Naamah, the lovely sister of Tubal-cain, led the angels astray with her beauty, and from her union with Shamdon sprang the devil Asmodeus. She was as shameless as all the other descendants of Cain, and as prone to bestial indulgences.(...)'.74

$\mathrm{Au}$ vers 779 Noemie explique donc son propre nom:

Por ce ai je nom Noemie

Que ja nul home n'escondie

Mais d'après quelle tradition? Dans l'exégèse chrétienne, le nom de la fille de Lamech est interprétée comme voluptas. De ce qui précède nous pouvons conclure que l'auteur de la Bible anonyme a dû puiser dans des sources juives pour son portrait de Noemie. Si nous pouvons rendre compte des détails concernant sa conduite, nous n'avons trouvé nulle part un modèle pour le monologue que prononce la jeune fille aux vers 777-786.

La Bible anonyme est la seule adaptation biblique à insister sur le personnage de Noemie; de plus, l'auteur lui attribue un 'métier' infâme. Là où la plupart des textes

\footnotetext{
${ }^{66} P L 198,1079$.

67 Voir Fillion (1921-1924) ad Gn IV, 22. Voir ci-dessus [BH] et [MVT].

68 Ginzberg I, p. 65.

69 Ginzberg, V, 143, 148.

70 Ginzberg I, 123-124.

71 Ginzberg I, 118.

72 Ginzberg V, 147-148. Voir aussi Gn VI, 1-2, où les 'filii Dei' sont à interpréter comme les descendants de Seth (Fillion, ad Gn VI, 1-2).

73 Ginzberg V, 143.

74 Ginzberg I, 150-151. Istehar refusait de se laisser séduire par un autre ange déchu, Shemhazai, qui apprenait aux hommes l'usage d'armes, de bijoux, de cosmétique et les sciences de l'exorcisme. De divination et d'astrologie (Ginzberg I, 125 et V, 172). Lilith et Naamah seraient aussi les deux femmes contestant la maternité d'un enfant devant Salomon (VI, 284). Voir aussi S. Baring-Gould (1881, p. 98).
} 
médiévaux passent sous silence le rôle de Noema, il est intéressant de noter que le Mistere du Vieil Testament lui prête un rôle important, et une voix $!^{75}$

Nous avons constaté que [MVT] relate en détail la bigamie de Lamech; comme on le verra par la suite, il raconte également la légende selon laquelle Lamech tua Caïn.

Mais ce qui frappe surtout c'est que [MVT], comme la Bible anonyme, présente Noemie comme une fille légère. Lorsque les 'filii Dei', les descendants de Seth, commencent à s'unir aux femmes de la race de Caïn, Caynam ${ }^{76}$ affirme: $^{\circ}$

Or en la ligne de Cayn

I a de belles femmes,gentes,

Tressubtilles et excellentes,

Comme Noema la gentille,

La quelle a esté si subtille

D'avoir trouvé l'art de tisture

Pour draper et faire vesteure,

Qui est ung tresgrant bien pour eulx;

Par quoy, je dy que par droicture

Et sans commettre aucune injure

Nous en pourrons estre amoureux. (4979-4989)

et prie Noema d'amour v.5011 ss.

Noema, fille gracieuse,

Tressubtille et ingenieuse,

Requerir te viens doulecement

Que tu soyez mon amoureuse;

Tu ne fus jamais si eureuse

Que d'avoir mon atouchement (5012-5017)

Noema répond:

Vous parlez amoureusement,

Doulx Caynam, je suis contrainte

De vous aymer, combien qu'en crainte,

Car, ainsi que j'ay entendu,

Il nous est de Adam deffendu

Nostre lignaige entremesler. (5018-5023)

Irard, fils de Caïn, affirme qu'il n'y a aucun danger à 's'entremesler'. Noema: 'J'en suis d'acord;/A ce n'y aura point d'offence'. (5037-5038). Sella, la mère de Noemie, ayant appris de la jeune fille que les fils de Seth 'ont mis leur cueur a nous aymer'(5333), dit à sa fille:

En effect, si vous estes prestes

D’acomplir le charnel desir

\footnotetext{
75 Dans la Noordnederlandse Historiebijbel c'est Noema qui prononce la 'prophétie' que Lamech adresse à ses femmes dans Gn IV, 23-24 (éd. M.K.A. van den Berg, Hilversum 1998, p. 233, sans commentaire de l'éditeur).

76 Fils d'Enos qui est le fils de Seth (Gn V, 9).
} 
Avec eulx pour vostre plaisir,

Ainsi que vous j'en suis contente. (5338-5341).

Ensuite c'est Malalael qui la prie d'amour: 'Se vueil passer temps avec vous,/ Vous desplaira il point?', prière à laquelle Noema répond: 'Nenny./De beaulté estes si muny/Que je ne vous puis escondire' (5346-5349). Mathusael, descendant de Caïn dit des filles de celui-ci:

(...) elles sont belles

Et ne demandent autre chose

Fors que avec elles on repose

Par desordonnée volupté (5378-5381

Finalement Jareth, descendant de Seth, dit à Noema: 'Noema, faisons bonne chere' (5580), à quoi elle répond: 'Jaret, mon amy par amour,/Je suis courcée, l'eure et le jour/ Que ne vous tiens entre mes bras'(5603-5605).

\section{(e) Lamech tue un homme}

Comme nous l'avons constaté ci-dessus, le vir tué par Lamech est identifié dans la plupart des adaptations bibliques avec Caïn. Or, dans notre texte, l'auteur, en parlant de Noemie, dit seulement:

Lamec, ce m'est vis, fuit ses peres

788 Et por li ocist un suen frere

De même dans $[\mathrm{A}]$ et $[\mathrm{M}]$, le dernier ajoutant après le vers 788:

.Ij. femmes avoit cil chaitis

Qui son frere avoit occis.

Si l'ajout dans $[\mathrm{M}]$ peut prêter à confusion, il est clair que notre texte désigne par ce frere le frère de Noemie, Tubalcain, qui, selon la légende, aurait été tué par son propre père. ${ }^{77}$ L'affirmation que Lamech tua Tubalcain 'pour' ou 'à cause de' Noemie (por li) reste tout à fait énigmatique. Nous avons vu que les autres adaptations bibliques ne donnent aucune interprétation de l'adolescentulum de Gn IV, 23. À part notre Bible anonyme, le seul texte à mentionner le frère de Noemie est le $[\mathrm{MVT}]$ où Lamech tue son fils Tubalcaim. ${ }^{78}$

Résumons les rapports entre la Bible anonyme et [MVT]:

- insistance sur la bigamie de Lamech

- Tubalcain fait des armes pour combattre les gens ${ }^{79}$

- le rôle de Noemie comme 'femme facile / légère'; ses discussions

- Lamech tue son propre fils

\footnotetext{
77 Voir J. P. Migne (1856, I, pp. 343-344); S. Baring-Gould (1881, p. 97). Reiss (1972, pp. 39-40). Murdoch (2003, pp. 72-74). Smeets (1967, I, pp. 31-32). L. R. Muir (1995, pp. 71); 208-210.

78 Rothschildt (1878), v. 4785.

79 Pas dans les adaptations bibliques, sauf dans [BH] (=Flave Josèphe).
} 
Relevons finalement les rapports plus ou moins textuels:

[MVT]: J'en suis d'acord;/A ce n'y aura point d'offence (5037-5038).

Bible anonyme: Je ne cuit riens meffaire a Dieu (782)

[MVT]: En effect, si vous estes prestes

D'acomplir le charnel desir (5338-5339)

Bible anonyme: Je suis preste si com je doi (783)

[MVT]: Nenny.

De beaulté estes si muny

Que je ne vous puis escondire (5346-49)

Bible anonyme: Nul n'escondi qui la pria (774); Que ja nul home n'escondie (780)

[MVT]: Et ne demandent autre chose

Fors que avec elles on repose

Par desordonnée volupté (5378-5381

Bible anonyme: N'ot en li nulle contenance (765); Por ce ai je nom Noemie [=voluptas $]$ (779)

\section{Conclusion}

Comme en témoignent la plainte de Jacob sur Joseph (vv. 2529-2546) et celle de la reine éprise du jeune homme (vv. 2590-2640), ${ }^{80}$ l'auteur de la Bible anonyme a un goût pour les monologues, qui confèrent un caractère dramatique à son texte. Il en est de même, par exemple, pour la 'Bible' d'Herman de Valenciennes qui, selon A. de Mandach consiste en 'twenty-two 'mystery plays' répartis sur trois cycles. ${ }^{81} \mathrm{Le}$ monologue de Noemie se distingue des autres monologues dans la mesure où ceuxci se rattachent toujours directement au texte biblique. Le discours de Noemie, et le cadre dans lequel il se situe, semblent parfaitement s'accorder avec la matière utilisée par les fatistes, qui exploitent une multitude de sources pour composer leurs ouvrages dans lesquels 'chaque situation, indiquée souvent d'un mot par la Bible, est développée en longs dialogues avec une grande abondance d'imagination dramatique'. ${ }^{82}$

L'histoire de Lamech a suscité l'intérêt de beaucoup d'auteurs médiévaux et de critiques modernes. Les uns et les autres mettent surtout l'accent sur le rôle de Lamech comme bigame et comme l'assassin de Cain; les inventions des trois fils de Lamech ont également donné lieu à des commentaires. Ce qui frappe c'est que ni les

\footnotetext{
${ }^{80}$ La plainte de la reine dans la Bible anonyme n'a pas de rapports avec celle de la reine dans la Bible de Malkaraume, dont J.-R. Smeets a montré la dépendance directe de la plainte de Médée dans les Métamorphoses d'Ovide. Voir J.-R. Smeets (1975) et son édition de la Bible de Jehan Malkaraume (1978, I, pp. 265-266).

81 A. de Mandach (1993). Voir J.R. Smeets, 'Herman de Valenciennes' dans GRLMA VI, 1, pp. 52-53.

${ }^{82}$ L. Petit de Julleville (1880, II, p. 363).
} 
auteurs des adaptations bibliques, ni les chercheurs modernes, ${ }^{83}$ même ceux qui ont commenté l'histoire de Lamech dans le Mistère du Viel Testament, ${ }^{84}$ n'ont prêté attention à la fille de Lamech, Noemie, qui n'est pas seulement l'objet de commentaires patristiques et juives, mais qui joue aussi un rôle considérable dans ce mystère du milieu du $\mathrm{XV}^{\mathrm{e}}$ siècle.

Si l'histoire des dix fils de Lamech reste, pour autant que nous sachons, un récit unique parmi les traductions-adaptations de la Bible en ancien français, vu ce qui précède par rapport à la présentation de Noemie, il serait tentant de croire à l'existence d'une source commune à l'histoire de Lamech dans notre Bible et à celle dans [MVT]. Source pourtant que l'on ne saurait identifier pour autant. Comme l'affirme Petit de Julleville: 'Indiquer d'une façon complète les sources multiples où les auteurs des mystères ont puisé le fond de leur oeuvre, serait une tâche assez grande pour remplir un livre'. ${ }^{85} \mathrm{Si}$ on réussit parfois à identifier une source profane dans les adaptations bibliques, ${ }^{86}$ un livre ne suffirait pas pour décrire les emprunts qu'elles font à la matière exégétique et apocryphe, chrétienne ou juive.

Avec la présente étude, nous espérons toutefois contribuer à la compréhension du travail des traducteurs bibliques, qui ne cesse de piquer notre curiosité.

Open Access This article is distributed under the terms of the Creative Commons Attribution Noncommercial License which permits any noncommercial use, distribution, and reproduction in any medium, provided the original author(s) and source are credited.

\section{Ouvrages cités}

Baring-Gould, S. (1881). Old Testament Patriarchs and Prophets, New York, repr. La Vergne USA 2010. Beichner, P. E. (Ed.). (1965). Petrus Riga, Aurora. Biblia versificata (2 vols). Notre Dame Indiana: University of Notre Dame Press.

Blatt, F. (Éd.). (1958). Antiquités juives. The Latin Josephus: Introduction and Text, The Antiquities, Acta Jutlandica 30, 1; Humanistic Series 44, Aarhus \& Copenhague.

Boers, W. (Ed.). (2002). La Genèse d'Evrat. Brive la Gaillarde: Ver luisant.

Bonnard, J. (1884). Les Traductions de la Bible en vers français au Moyen Age. Paris: Imprimerie nationale.

Colunga, A. O. P., \& Turrado, L. (Eds.). (1982). Biblia sacra iuxta Vulgatam clementinam, sexta editio. Madrid: Biblioteca de Autores Cristianos.

David, J. (1858-1859). Jacob van Maerlant, Rymbybel (4 vols), Brussel: Hayez.

de Mandach, A. (1993). The Creation of Herman de Valenciennes: An unpublished Anglo-Norman mystery play of the 12th century. Anglo-Norman Anniversary Essays, ed. I. Short (pp. 251-272). London: Anglo Norman Text Society.

Douhet, J. (1854). Dictionnaire des Mystères, ou collection générale des mystères, moralités, rites figurés et cérémonies singulières (...). Paris: Ateliers catholiques.

Fillion, L.Cl. (1921-1924). La Sainte Bible commentée d'après la Vulgate et les textes originaux, Paris: Letouzey et Ané.

\footnotetext{
${ }_{83} \mathrm{Ni}$ Bonnard (1884), ni Smeets (GRLMA VI/1, pp. 48-57) n'ont signalé le récit curieux des enfants de Lamech.

84 Ainsi Reiss (1972), Rothschild (1878), Murdoch (2003).

85 Les Mystères, I, pp. 202-203. Nous savons pourtant que le fatiste a mis à profit la Penitance of Adam pour la partie qui concerne Adam et Ève (ibid. p. 205). Voir aussi Rothschild (1878), pp. ix-xij. Voir G.A. Runnalls (1998, pp. 279-85).

${ }^{86}$ Voir Un fragment de la Genèse en vers (Szirmai 2005, p. 111, n. 325).
} 
Gibson, M.T. \& Froehlich, K. (Intr.) (1992). Biblia latina cum Glossa Ordinaria. Facsimile Reprint of the Editio Princeps, Adolph Rusch of Strasbourg 1480/81. Turnhout: Brepols.

Ginzberg, L. (1911-1938). The Legends of the Jews. Philadelphia: The Jewish Publication Society of America.

Godefroy, F. (1881-1902). Dictionnaire de l'ancienne langue française (10 vols), Paris: Vieweg.

Harf-Lancner, L. (1984). Les Fées au Moyen Âge. Paris: Honoré Champion.

Loh, H. (Ed.). (1912). Histoires tirées de l'Ancien Testament. Münster: Universitätsdruckerei Johannes Bredt.

Mazière, F. [Trad.). (1975) Le Livre d'Enoch. Paris: Laffont.

Mellinkoff, R. (1981). The Mark of Cain. Berkeley: University of California Press.

Michel, B. (Éd.). (2004). Guiart Des Moulins, Bible historiale. Thèse de doctorat Nouveau Régime, Univ. De Bourgogne.

Migne, J. P. (Ed.). (1850). Saint Isidore, Quaestiones in vetus Testamentum. PL 83. Paris: Ateliers catholiques.

Migne, J. P. (Ed.). (1851). Raban Maur, Commentariorum in Genesim. PL 107. Paris: Ateliers catholiques.

Migne, J.P. (Ed.). (1855, repr. 1980). Petrus Comestor, Historia Scholastica. PL 198. Paris: Ateliers catholiques.

Migne, J. P. (1856). Dictionnaire des apocryphes. Paris: Ateliers catholiques.

Morey, J. H. (1993). Peter Comestor, Biblical Paraphrase, and the Medieval popular Bible. Speculum, $68(1), 6-35$.

Muir, L. R. (1995). The biblical drama of medieval Europe. Cambridge: Cambridge University Press.

Murdoch, B. (2003). The medieval popular Bible. Oxford: Brewer.

Nobel, P. (Ed.). (1996). Poème anglo-normand sur l'Ancien Testament (2 vols). Paris: Champion.

Nobel, P. (Ed.). (2006). La Bible d'Acre. Genèse et Exode. Besançon: Presses universitaires de FrancheComté.

Petit de Julleville, L. (1880). Les Mystères. Paris: Repr. Elibron Classics 2005.

Quereuil, M. (Ed.). (1988). La Bible française du XIII ${ }^{e}$ siècle. Genève: Droz.

Reiss, E. (1972). The story of Lamech and its place in medieval drama. Journal of Medieval and Renaissance Studies, 2, 35-48.

Rothschild, J. de. (Éd.). (1878-1891). Le Mistère du Vieil Testament (6 vols). Paris: Firmin Didot.

Runnalls, G. A. (1998). Études sur les mystères. Paris: Champion.

Santamarta del Rio, S. y. M., \& Fuertes Lanero, M. (Eds.). (1978). Saint Augustin, De Civitate Dei. Madrid: Biblioteca de autores cristianos.

Schmitt, J.-Cl. (1988). Les superstitions. Histoire de la France religieuse, Le Goff. J. \& Remond, R. (Red.), I, 4. Paris: Seuil.

Smeets, J.-R. (Ed.). (1967). La Bible de Macé de La Charité. Genèse, Exode. Leiden: Universitaire Pers.

Smeets, J. R. (1968). Les traductions, adaptations et paraphrases de la Bible en vers, Grundriss der Romanischen Literaturen des Mitelalters VI/1, 48-57. Heidelberg: Carl Winter.

Smeets, J.-R. (1975). Le monologue de la roïne dans la 'Bible' de Malkaraume. Mélanges de linguistique et de littérature offerts à Lein Geschiere (pp. 11-24). Amsterdam: Rodopi.

Smeets, J.-R. (Ed.). (1978). La Bible de Jehan Malkaraume (2 vols). Assen/Amsterdam: van Gorcum. Sylwan, A. (Ed.). (2005). Petri Comestoris Scolastica Historia, Liber genesis. Turnhout: Brepols.

Szirmai, J. C. (Ed.). (1985). La Bible anonyme du Ms. Paris B.N.F. fr. 763. Amsterdam: Rodopi.

Szirmai, J. C. (Ed.). (2005). Un fragment de la Genèse en vers (fin XIIIe-début XIVe siècle). Genève: Droz 2005.

Tobler, A. \& Lommatzsch, E. (1925). Altfranzösisches Wörterbuch, Berlin: Weidmannsche Buchhandlung. van den Berg, M. K. A. (Ed.). (1998). De Noordnederlandse Historiebijbel. Hilversum: Verloren.

van der Mark, A. (2005). La notion de 'nigremance' dans les textes littéraires du Moyen Âge, Etude sur un ensemble de pratiques magiques. Leiden: Mémoire de Master, Universiteit Leiden.

van Hamel, A.-G. (Ed.). (1905). Les lamentations de Matheolus et le Livre de Leesce de Jehan le Fèvre de Ressons. Paris: Bouillon.

Verduin, C.J. (2003). La recherche de GIIOHARGIIIVS, 'Tubalcain, Jubal \& Pythagoras', www.leidenuniv. $\mathrm{nl} / \mathrm{fsw} /$ verduin/ghio/sourchro.htm.

Vigouroux, F. (1895-1912). Dictionnaire de la Bible (5 vols), Paris: Letouzey et Ané. 